\title{
Metabolic Networks Underlying Cognitive Reserve in Prodromal Alzheimer Disease: A European Alzheimer Disease Consortium Project
}

\author{
Silvia Morbelli ${ }^{1}$, Robert Perneczky ${ }^{2,3}$, Alexander Drzezga ${ }^{4,5}$, Giovanni B. Frisoni ${ }^{6}$, Anna Caroli ${ }^{6,7}$, Bart N.M. van Berckel ${ }^{8}$, \\ Rik Ossenkoppele ${ }^{8}$, Eric Guedj ${ }^{9}$, Mira Didic ${ }^{10}$, Andrea Brugnolo ${ }^{11}$, Mehrdad Naseri ${ }^{1}$, Gianmario Sambuceti ${ }^{1}$, \\ Marco Pagani ${ }^{12,13}$, and Flavio Nobili ${ }^{11}$ \\ ${ }^{I}$ Nuclear Medicine, Department of Internal Medicine, IRCCS AOU San Martino-IST, University of Genoa, Genoa, Italy; ${ }^{2}$ Department \\ of Psychiatry and Psychotherapy, Technische Universität, Munich, Germany; ${ }^{3}$ Neuroepidemiology and Ageing Research Unit, School \\ of Public Health, Faculty of Medicine, Imperial College of Science, Technology, and Medicine, London, United Kingdom; \\ ${ }^{4}$ Department of Nuclear Medicine, Technische Universität, Munich, Germany; ${ }^{5}$ Department of Nuclear Medicine, University of \\ Cologne, Cologne, Germany; ${ }^{6}$ LENITEM Laboratory of Epidemiology and Neuroimaging-IRCCS S. Giovanni di Dio-FBF, Brescia, \\ Italy; ${ }^{7}$ Medical Imaging Unit, Biomedical Engineering Department, Mario Negri Institute, Bergamo, Italy; ${ }^{8}$ Department of Nuclear \\ Medicine \& PET Research, VU University Medical Center, Amsterdam, The Netherlands; ${ }^{9}$ Aix-Marseille University, CERIMED \\ and CNRS, Marseille, France; ${ }^{10}$ APHM, CHU Timone, Service de Neurologie et Neuropsychologie, Aix-Marseille University, \\ INSERM U 1106, Marseille, France; ${ }^{11}$ Clinical Neurophysiology, Department of Neuroscience, Ophthalmology, and Genetics, \\ IRCCS AOU San Martino-IST, University of Genoa, Genoa, Italy; ${ }^{12}$ Institute of Cognitive Sciences and Technologies, CNR, Rome, \\ Italy; and ${ }^{13}$ Department of Nuclear Medicine, Karolinska Hospital, Stockholm, Sweden
}

This project aimed to investigate the metabolic basis for resilience to neurodegeneration (cognitive reserve) in highly educated patients with prodromal Alzheimer disease (AD). Methods: Sixty-four patients with amnestic mild cognitive impairment who later converted to $A D$ dementia during follow-up, and 90 controls, underwent brain ${ }^{18} \mathrm{~F}$-FDG PET. Both groups were divided into a poorly educated subgroup (42 controls and 36 prodromal $A D$ patients) and a highly educated subgroup (48 controls and 28 prodromal AD patients). Brain metabolism was first compared between education-matched groups of patients and controls. Then, metabolism was compared between highly and poorly educated prodromal $A D$ patients in both directions to identify regions of high education-related metabolic depression and compensation. The clusters of significant depression and compensation were further used as volumetric regions of interest (ROIs) in a brain interregional correlation analysis in each prodromal $A D$ subgroup to explore metabolic connectivity. All analyses were performed by means of SPM8 $(P<0.001$ uncorrected at peak level, $P<0.05$ false discovery rate-corrected at cluster level; age, sex, Mini-Mental State Examination score, and center as nuisance). Results: Highly educated prodromal $A D$ patients showed more severe hypometabolism than poorly educated prodromal $A D$ patients in the left inferior and middle temporal gyri and the left middle occipital gyrus (ROI depression). Conversely, they showed relative hypermetabolism in the right inferior, middle, and superior frontal gyri (ROI compensation). The sites of compensation, mainly corresponding to the right dorsolateral prefrontal cortex (DLFC), showed wide metabolic correlations

Received Sep. 21, 2012; revision accepted Jan. 4, 2013.

For correspondence or reprints contact: Silvia Morbelli, San Martino Hospital, Largo R. Benzi 10, 16132 Genoa, Italy.

E-mail: silviadaniela.morbelli@hsanmartino.it

Published online Apr. 16, 2013.

COPYRIGHT @ 2013 by the Society of Nuclear Medicine and Molecular Imaging, Inc. with several cortical areas in both hemispheres (frontotemporal cortex, parahippocampal gyrus, and precuneus) in highly educated prodromal AD patients but not in poorly educated prodromal $A D$ patients. To provide evidence on whether these metabolic correlations represent preservation of the physiologic networks of highly educated control subjects (neural reserve) or rather the recruitment of alternative networks (neural compensation), or a combination of the two, we performed metabolic connectivity analysis of the DLFC in highly educated controls as well. The correlation sites of right DLFC partly overlapped those of highly educated prodromal $A D$ patients but were less extended. Conclusion: The present findings suggest that highly educated prodromal AD patients can cope better with the disease thanks to neural reserve but also to the recruitment of compensatory neural networks in which the right DLFC plays a key role.

Key Words: Alzheimer's disease; mild cognitive impairment; cognitive reserve; positron emission tomography; metabolic connectivity

J Nucl Med 2013; 54:894-902

DOI: 10.2967/jnumed.112.113928

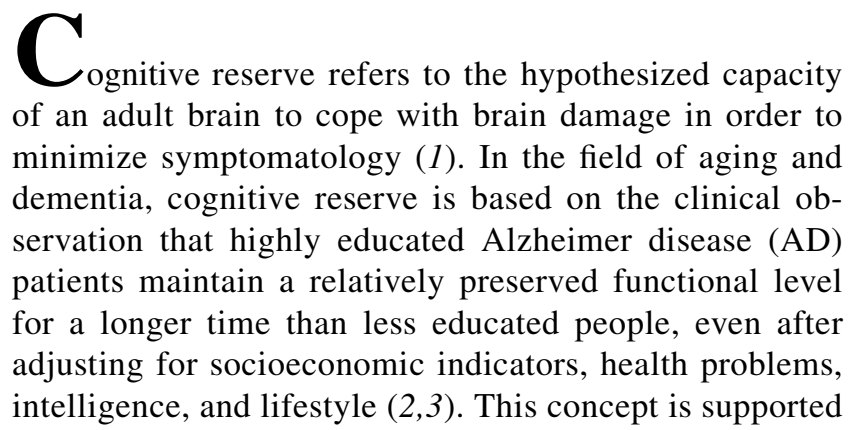


by frequent discrepancies between measured pathology and cognitive decline $(4,5)$. It has been shown that about $20 \%$ of elderly people who are cognitively normal before death have sufficient postmortem pathology to meet neuropathologic criteria for $\mathrm{AD}(6,7)$, and such a discrepancy could be due to cognitive reserve.

A critical question for the understanding of cognitive reserve is to clarify the brain adaptation mechanisms underlying this concept that otherwise remains an abstract conjecture. The neuropsychologic approach uses several variables as markers of, or proxies for, cognitive reserve (i.e., education, occupational complexity, extent of intellectual activities during leisure time, and neuropsychologic tests) (8). Neuropsychologic tests are sensitive to brain injury and dysfunction, but they yield only indirect evidence of brain networking and lack precise anatomic localization (9).

Functional neuroimaging has the potential to contribute to the understanding of these adaptive mechanisms of the brain. That is, given a particular level of imaging-assessed brain damage, cognitive reserve could hypothetically be defined as the difference between an individual's expected and actual cognitive performance. Indeed, neuroimaging measurements reflect more closely the pathophysiologic condition of a given patient whereas neuropsychology is more directly influenced by the effect of cognitive reserve itself.

Moreover, functional imaging may help disclose the compensatory circuitry that allows a subject with more efficient brain reserve to cope with cognitive tasks despite the presence of degenerative pathology. Following this hypothesis, the presence of cognitive reserve has been specifically investigated by means of functional MR imaging, both perfusion and metabolism PET, and, more recently, amyloid PET imaging (10). Functional MR imaging and perfusion $\left(\mathrm{H}_{2}{ }^{15} \mathrm{O}\right)$ PET activation studies have provided information on compensatory functional networks related to a specific task (11). On the other hand, resting-state functional MR imaging studies allowed the identification of different regions of brain activation during psychosensorial resting (12).

These imaging studies have been performed on healthy aging individuals with mild cognitive impairment (MCI) (13) and in several neurodegenerative diseases, including $\mathrm{AD}(12,14)$. In the field of dementia, most studies on cognitive reserve have included healthy elderly and AD patients but rarely (13) subjects at prodromal stages of the disease.

As far as resting-state metabolic aspects of brain reserve are concerned, previous ${ }^{18} \mathrm{~F}-\mathrm{FDG}$ PET studies indirectly suggested the presence of cognitive reserve in $\mathrm{AD}$ patients through the demonstration of an inverse relationship between glucose metabolism and education/occupation $(13,14)$ or intellectual ability (15). However, evidence of more extended or preserved metabolic connectivity in subjects with a higher educational level is still lacking, especially in the prodromal stage of $\mathrm{AD}$, when both pharmacologic and training interventions are more likely to be successful.

The aim of this study was to investigate the metabolic bases of cognitive reserve in patients with prodromal $\mathrm{AD}$ and high education. In particular, we aimed at directly demonstrating the presence of more severe brain glucose hypometabolism in a group of highly educated patients with prodromal $\mathrm{AD}$ than in severity-matched poorly educated patients. Then, our hypothesis was that cognitive reserve in highly educated patients results from more widespread metabolic connections of relatively preserved brain regions. We applied the method of interregional correlation analysis based on statistical parametric mapping (SPM) data, which has been successfully used to investigate metabolic connectivity both in overt (16) and in prodromal (17) AD.

\section{MATERIALS AND METHODS}

\section{Subjects}

The ${ }^{18}$ F-FDG PET project of the European Alzheimer Disease Consortium (http://www.eadc.info) is aimed at joining together ${ }^{18}$ F-FDG PET scans with corresponding clinical and neuropsychologic data from patients with MCI and cognitively normal controls.

Patients with amnestic MCI, with or without impairment in other cognitive domains, and healthy controls were enrolled. Dementia was excluded by clinical interviews with patients and caregivers exploring significant impairment in activities of daily living and by means of the Clinical Dementia Rating scale (18), scoring 0.5 in all patients. General cognition was assessed with the Mini-Mental State Examination (MMSE) in all centers.

All subjects underwent rating scales for depression and neuropsychiatric symptoms according to the routine in use in each center. Neuropsychologic tests were administered in the domains of memory, language, executive function, attention, and visuoconstruction, according to the routine of each center, to define the amnestic MCI syndrome, following Petersen's criteria (19). Raw scores were converted to age-, education-, and sex-corrected $z$ scores according to each center's locally collected or normative data, which vary among countries and languages. Impairment was defined as a $z$ score of -1.5 or lower. Patients with impairment in the memory domain only (single-domain amnestic MCI) or with impairment in the memory domain plus impairment in nonmemory domains (multidomain amnestic MCI) were included.

The healthy controls agreed to participate as volunteers, and the only inclusion criterion was that they be in the same age range as the patients. They were judged to be in good health by general medical and neurologic examinations and by routine blood and urine assays. Cognitive health was established in each center by means of the same interview and test battery as that in use for amnestic MCI patients. Only subjects with an MMSE score of more than 26 and a Clinical Dementia Rating score of 0 were included.

Further details about inclusion and exclusion criteria for both amnestic MCI patients and controls have been listed elsewhere (17) and are included as supplemental material (available online at http://jnm.snmjournals.org).

Patients and controls were regularly followed up to detect those developing dementia. Patients were carefully treated for systemic comorbidity; drugs known to depress brain synaptic transmission, such as benzodiazepines and tricyclic antidepressants, were slowly tapered and then withdrawn, when possible. None of the patients was taking antidementia medication at the time of either PET scan or during the follow-up period. The study was approved by the local Ethics Committees, and all the recruited subjects provided written informed consent. 
The present work concerns the data of 186 patients with amnestic MCI and 90 controls enrolled and followed up until December 2011. The proportion of patients and controls was roughly comparable among centers to balance for the impact of different scanners.

During a mean follow-up of about 2 y, 64 of the 186 amnestic MCI patients progressed to $\mathrm{AD}$ dementia (referred to in this paper as prodromal AD patients: 31 men and 33 women; mean age, $72.0 \pm 8.2$ y; years of education, $11.0 \pm 4.6$; mean baseline MMSE score, $27.0 \pm 1.6$; mean follow-up time, $22.6 \pm 16.0 \mathrm{mo}$ ). Diagnosis of dementia of the $\mathrm{AD}$ type was established according to the criteria of the National Institute of Neurological and Communicative Disorders and Stroke and the Alzheimer's Disease and Related Disorders Association (20) and according to the DSM-IVR criteria (21). To investigate the pathophysiology of brain cognitive reserve, both prodromal $\mathrm{AD}$ and control groups were further divided into low-education (LE) and high-education (HE) subgroups according to the median number of years of schooling of the whole group. This cutoff (12 y of education) allowed the following subgroups to be obtained: HE control $(n=48)$, LE control $(n=42)$, HE prodromal $\mathrm{AD}(n=28)$, and LE prodromal $\mathrm{AD}(n=36)$ (Table 1$)$.

This median of $12 \mathrm{y}$ is close to the boundary between high-level and medium- to low-level education in the 4 countries participating in the study, that is, 9-10 y in Germany, $11 \mathrm{y}$ in The Netherlands, $12 \mathrm{y}$ in France, and $13 \mathrm{y}$ in Italy. Supplemental Figure 1 shows the distribution of classes of years of education. Table 2 reports the baseline neuropsychologic $z$ scores of the patient groups. Neither the MMSE score nor scores on neuropsychologic tests significantly differed between the 2 prodromal AD subgroups, thus allowing the investigation of metabolic impairment between differently educated prodromal AD patients with a comparable severity of cognitive impairment.

\section{PET Procedures}

${ }^{18}$ F-FDG PET was performed, according to European Association of Nuclear Medicine guidelines, within 2 mo from the baseline clinical-neuropsychologic examination (22). Technical details of the scanners used in the different centers are reported in Supplemental Table 1. DICOM files were exported and converted to Analyze format.

\section{Image Analysis}

All preprocessing and statistical analysis steps were performed using the SPM package (version 8; Wellcome Department of Cognitive Neurology) (23) implemented in Matlab, version 6.5 (MathWorks). To avoid inconsistencies deriving from the use of the default SPM brain $\mathrm{H}_{2}{ }^{15} \mathrm{O}$ template (24), PET scans were normalized using a customized brain ${ }^{18} \mathrm{~F}$-FDG PET template, obtained from brain PET and MR imaging scans of 27 healthy subjects as detailed elsewhere (17). This setting is absolutely similar to the SPM default (the template available for normalization is based on controls acquired in a single center, which is, by definition, different from the center where the studied PET scans were acquired). However, even if a gold standard has not been identified, we cannot exclude the possibility that the inclusion of controls from other centers could have influenced the results of the analyses. After template editing, all brain PET scans were subjected to affine and nonlinear spatial normalization into the stereotaxic space of the Montreal Neurological Institute through the study-customized ${ }^{18}$ F-FDG template using SPM. The spatially normalized sets of images were then smoothed with a 10-mm isotropic gaussian filter to blur individual variations in gyral anatomy and to increase the signal-to-noise ratio.

\section{Comparison of HE and LE Prodromal AD Groups with HE and LE Control Groups}

This step assessed whether, in comparison to age- and education-matched controls, HE prodromal AD patients effectively had more severe hypometabolism than LE prodromal AD patients who had similar global cognitive impairment. To this aim, brain PET scans from prodromal AD subgroups were compared with those from the age- and education-matched control groups on a voxel-by-voxel basis. The following comparisons were performed (2-sample $t$ test): HE control $>$ HE prodromal AD and LE control > LE prodromal AD.

The significance of identified regions was established at a $P$ value of less than 0.05 , corrected for multiple comparisons with the false discovery rate (FDR) option at both peak and cluster level. Only clusters containing more than 50 voxels were considered to be significant.

In this preliminary step, more severe and extended hypometabolism was indeed present in HE prodromal AD patients. Clusters of hypometabolism in HE prodromal AD patients were present in the left middle occipital gyrus, bilaterally in the superior and middle temporal gyri, and in wide regions of the posterior parietal cortex in both hemispheres, in addition to bilaterally in the precuneus and posterior cingulate cortex (Fig. 1 and Supplemental Table 2). Therefore, in the following analyses we focused on investigating brain regions, and then metabolic networks, that allow HE prodromal AD patients to maintain a level of global cognitive functioning similar to that of LE prodromal AD patients, despite more severe AD-related hypometabolism.

TABLE 1

Patient and Control Characteristics

\begin{tabular}{lccc}
\hline Characteristic & HE control $(n=48)$ & LE control $(n=42)$ & HE prodromal AD $(n=28)$ \\
\hline Age $(y)$ & $68.6 \pm 6.5$ & $65.4 \pm 5.9$ & $71.0 \pm 8.3$ \\
Sex & 25 & 14 & 18 \\
Men & 23 & 28 & 10 \\
Women & $14.4 \pm 2.2$ & $7.8 \pm 1.9$ & $15.1 \pm 2.4$ \\
Education (y) & $16.1 \pm 18.2$ & $19.9 \pm 17.1$ & $24.3 \pm 18.0$ \\
Follow-up (mo) & $29.4 \pm 1.0$ & $29.1 \pm 1.4$ & $27.2 \pm 1.3$ \\
Baseline MMSE & $29.1 \pm 1.2$ & $29.0 \pm 1.6$ & $24.1 \pm 2.2$
\end{tabular}

Data are mean $\pm \mathrm{SD}$ 


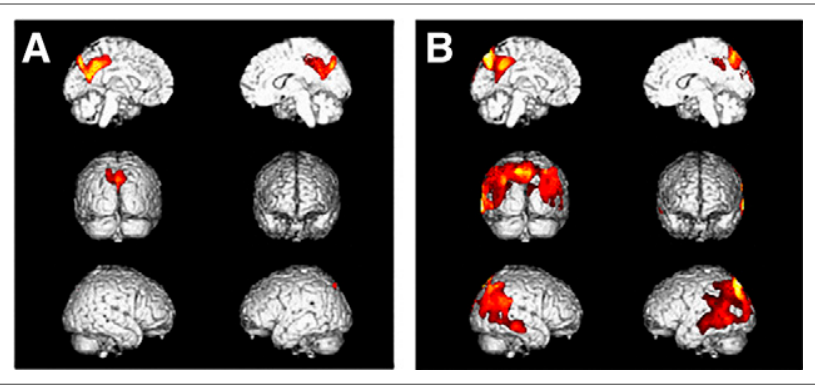

FIGURE 1. Comparison between LE (A) and HE (B) prodromal $A D$ patients and education-matched controls. Height significance threshold: $P<0.05$, corrected for multiple comparisons (false discovery rate), at both peak and cluster levels. Figure displays regions of significant difference, color-graded in terms of $z$ values. Talairach coordinates and further details are provided in Supplemental Table 2.

\section{Regions of Metabolic Depression and Compensation in Prodromal AD Subgroups}

To identify the presence, location, and extent of brain regions with either greater metabolic depression or greater compensation in $\mathrm{HE}$ than in LE prodromal AD patients, we directly compared the PET scans of the 2 subgroups in both directions (2-sample $t$ test: $\mathrm{LE}>\mathrm{HE}$ and $\mathrm{HE}>\mathrm{LE}$ ). These analyses were performed by applying all the SPM parameters used for the comparison with controls, except for the $P$ value, which was accepted as significant at peak level (uncorrected $P<0.001$ ).

These comparisons allowed identifying a cluster of metabolic depression and a cluster of metabolic compensation in the HE prodromal AD subgroup (the results are presented in Fig. 2 and Table 3 ). These 2 clusters were finally saved and used as a volumetric region of interest (ROI): 1. ROI depression (LE $>\mathrm{HE}$ ); 2. ROI compensation (HE $>$ LE), respectively.

In each patient, mean ${ }^{18} \mathrm{~F}$-FDG uptake within ROI depression and ROI compensation was computed using the SPM Mask subtool. Individual ROI ${ }^{18} \mathrm{~F}-\mathrm{FDG}$ uptake values were then normalized to the individual mean uptake in the cerebellum (weighted ${ }^{18} \mathrm{~F}-\mathrm{FDG}$ uptake).

\section{Metabolic Connectivity of ROI Depression and Compensation}

To evaluate the functional connectivity of ROI depression and ROI compensation in $\mathrm{HE}$ and $\mathrm{LE}$ prodromal $\mathrm{AD}$ patients, voxelwise interregional correlation analysis was performed using SPM according to the procedure validated by Lee et al. (25). Briefly, in both the HE and the LE prodromal AD groups, mean regional counts of both ROI depression and ROI compensation were used as covariates to find regions showing significant voxelwise correlations across subjects (multiple regression analysis). This option allows the voxelwise evaluation of the correlation between variables of interest and PET-assessed brain metabolism in each subgroup. SPM $t$ maps were displayed using an uncorrected $P$ value of less than 0.001 at peak level; all other SPM analysis details were the same as in the previous analyses. In all SPM analyses, age, sex, MMSE score, and center of belonging were included as nuisance variables.

\section{RESULTS}

\section{Regions of Metabolic Depression and Compensation in HE Prodromal AD Patients}

In the direct comparison between HE and LE prodromal $\mathrm{AD}$ patients, a more severe hypometabolism was detected in the HE group in the fusiform gyrus, inferior and middle temporal gyri, and middle occipital gyrus of the left hemisphere (ROI depression) (Fig. 2A). Conversely, relatively higher metabolic levels were found in the right inferior, middle, and superior frontal gyri in HE than in LE prodromal AD patients (ROI compensation) (Fig. 2B). Table 3 provides details on cortical regions, Brodmann areas (BAs), and $z$ scores.

\section{Interregional Correlation Analysis of ROI Depression}

In both the HE and the LE prodromal AD subgroups, ROI depression showed a pattern only of autocorrelation. In fact, this region was metabolically connected with the left occipital and temporal lobes. In LE prodromal AD, only ROI depression was also correlated with a small cluster in the right cuneus (Fig. 3). Table 4 provides details on cortical regions, BAs, and $z$ scores.

\section{Interregional Correlation Analysis of ROI Compensation}

ROI compensation showed wide metabolic correlations with several cortical areas in both hemispheres (frontotemporal cortex, parahippocampal gyrus, and precuneus) in HE prodromal AD patients (Fig. 4B) but was substantially only autocorrelated in LE prodromal AD patients (Fig. 4A). Table 5 provides details on cortical regions, BAs, and $z$ scores.

\section{Post Hoc Analysis}

Overall, these results suggest some explanations about how $\mathrm{HE}$ prodromal $\mathrm{AD}$ patients can cope better with a more severe AD-related hypometabolic involvement. However, the results do not indicate whether these metabolic networks represent only the preservation of the physiologic networks that can also be found in HE cognitively normal subjects or rather represent the recruitment of alternative neural networks to support cognitive function in the presence of disease-related damage elsewhere.

To further investigate this aspect, the metabolic connectivity of ROI compensation (i.e., right dorsolateral prefrontal cortex [DLFC]) was separately tested in HE controls and in LE controls. This post hoc analysis was performed using the same parameters as those adopted for the

TABLE 2

Baseline Neuropsychologic $z$ Scores of Patient Groups

\begin{tabular}{lcrc}
\hline \multicolumn{1}{c}{ Group } & HE prodromal & LE prodromal & \\
& $\mathrm{AD}(n=28)$ & $\mathrm{AD}(n=36)$ & $P$ \\
\hline Immediate recall & $-1.83 \pm 1.00$ & $-1.25 \pm 0.99$ & $\mathrm{NS}$ \\
Delayed recall & $-2.06 \pm 1.07$ & $-1.89 \pm 0.74$ & $\mathrm{NS}$ \\
Visuoconstruction & $-0.53 \pm 1.94$ & $-0.48 \pm 1.76$ & $\mathrm{NS}$ \\
Verbal fluency & $-0.16 \pm 1.06$ & $0.47 \pm 1.38$ & $\mathrm{NS}$ \\
Attention & $-0.51 \pm 2.55$ & $-0.61 \pm 2.01$ & $\mathrm{NS}$ \\
Executive function & $-1.35 \pm 2.08$ & $-1.42 \pm 1.10$ & $\mathrm{NS}$ \\
& & & \\
\hline NS $=$ not significant. & & & \\
Data are mean $\pm \mathrm{SD}$. & & & \\
\hline
\end{tabular}


FIGURE 2. Comparison between LE and HE prodromal AD patients. Height thresholds: uncorrected $P<0.001$ at peak level; $P<0.05$ FDR-corrected at cluster level. These 2 clusters were finally saved and used as ROls, one with ROI depression $(\mathrm{A}, \mathrm{LE}>\mathrm{HE})$ and another with ROI compensation (B, HE > LE). Talairach coordinates and further details are provided in Supplemental Table 2 and in Table 3.

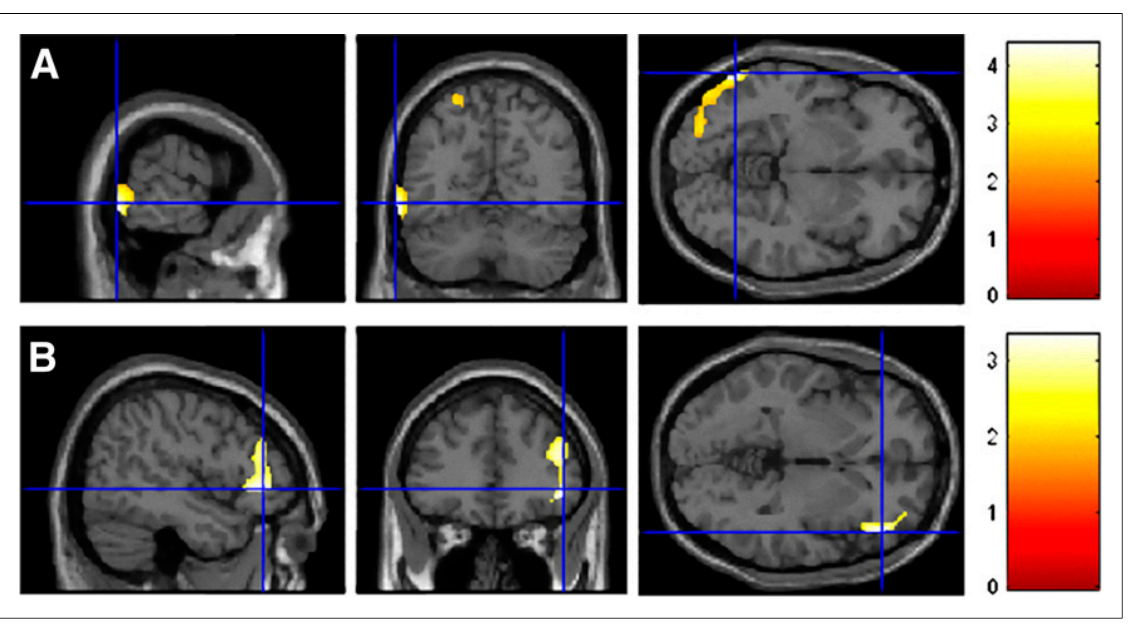

analysis in prodromal AD subgroups. The analysis showed that, although moderately less extended than in HE prodromal $\mathrm{AD}$ patients, the distribution of metabolic correlations in the right DLFC of HE controls is similar to that of HE prodromal AD patients (mainly including the frontal and temporal cortex, parahippocampal gyrus, and precuneus in both hemispheres) (Fig. 5B). By contrast, in LE controls the metabolic connectivity of the right DLFC was markedly less extended, as it was substantially autocorrelated and then just correlated with small clusters in the precuneus, occipital cortex, and limbic cortex mainly in the ipsilateral hemisphere (Fig. 5A). Figure 6 shows the point-by-point comparison of the distribution of the results of the voxelwise interregional correlation analysis of ROI compensation in HE prodromal $\mathrm{AD}$ patients and $\mathrm{HE}$ controls (details on cortical regions, BAs, and $z$ scores are provided in Supplemental Table 3).

\section{DISCUSSION}

This study investigated mechanisms of cognitive reserve by assessing the resting metabolic networks that enable HE prodromal AD patients to maintain a level of cognitive functioning similar to that of LE prodromal AD patients despite having greater brain metabolic impairment in areas typically affected by neurodegenerative changes in AD.

The main finding was that in these patients the greater associative and limbic posterior cortical metabolic dysfunc-

TABLE 3

Results of ${ }^{18} \mathrm{~F}-\mathrm{FDG}$ Brain PET Comparison Between HE and LE Prodromal AD Patients, with ROI Compensation

\begin{tabular}{|c|c|c|c|c|c|c|c|c|c|}
\hline \multirow[b]{3}{*}{ Analysis } & \multirow{2}{*}{\multicolumn{3}{|c|}{ Cluster level }} & \multicolumn{6}{|c|}{ Peak level } \\
\hline & & & & \multirow{2}{*}{$\begin{array}{l}\text { Maximum } \\
z \text { score }\end{array}$} & \multicolumn{3}{|c|}{$\begin{array}{l}\text { Talairach } \\
\text { coordinates }\end{array}$} & \multirow[b]{2}{*}{ Cortical region } & \multirow[b]{2}{*}{$\mathrm{BA}$} \\
\hline & Cluster extent & Corrected $P$ & Cortical region & & $x$ & $y$ & $z$ & & \\
\hline \multirow[t]{5}{*}{$\mathrm{LE}>\mathrm{HE}$} & 1,412 & 0.007 & L occipital & 4.4 & -42 & -74 & -10 & Fusiform gyrus & 19 \\
\hline & & & $\mathrm{L}$ temporal & 3.69 & -63 & -60 & -2 & Inferior temporal gyrus & 37 \\
\hline & & & $\mathrm{L}$ temporal & 3.25 & -50 & -73 & 11 & Middle temporal gyrus & 39 \\
\hline & & & L occipital & 3.21 & -46 & -81 & 10 & Middle occipital gyrus & 19 \\
\hline & & & L occipital & 3.06 & -26 & -84 & 1 & Middle occipital gyrus & 18 \\
\hline \multirow[t]{8}{*}{$\mathrm{HE}>\mathrm{LE}$} & 693 & 0.046 & $\mathrm{R}$ frontal & 3.34 & 48 & 35 & -3 & Inferior frontal gyrus & 47 \\
\hline & & & $R$ frontal & 3.15 & 50 & 29 & 2 & Inferior frontal gyrus & 45 \\
\hline & & & $\mathrm{R}$ frontal & 3.01 & 44 & 38 & 20 & Middle frontal gyrus & 46 \\
\hline & & & $R$ frontal & 2.8 & 50 & 32 & 8 & Inferior frontal gyrus & 46 \\
\hline & & & $\mathrm{R}$ frontal & 2.77 & 40 & 53 & 16 & Superior frontal gyrus & 10 \\
\hline & & & $R$ frontal & 2.68 & 34 & 13 & -12 & Inferior frontal gyrus & 13 \\
\hline & & & $\mathrm{R}$ frontal & 2.63 & 42 & 49 & 16 & Middle frontal gyrus & 46 \\
\hline & & & $R$ frontal & 2.5 & 30 & 9 & -17 & Inferior frontal gyrus & 47 \\
\hline
\end{tabular}

$P$ values of $<0.001$ (uncorrected at voxel level) and $<0.05$ (FDR-corrected for multiple comparisons at cluster level) were accepted as statistically significant. In cluster level section, reported for each statistically significant cluster are number of voxels, corrected $P$ value, and cortical region where cluster was found. In peak level section, reported for each significant cluster are $z$ score, peak coordinates, corresponding cortical region, and BA. 


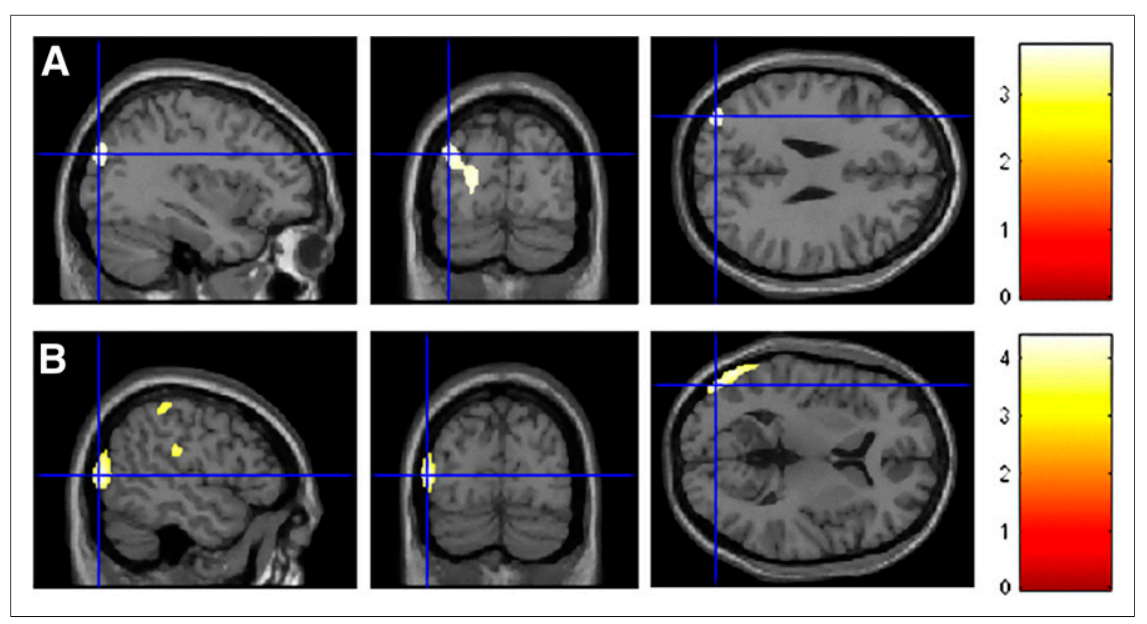

FIGURE 3. Voxelwise interregional correlation analysis of ROI depression in LE (A) and $\mathrm{HE}(\mathrm{B})$ prodromal $\mathrm{AD}$ patients. Talairach coordinates and further details are provided in Table 4. tion is counterbalanced by a relatively preserved metabolism in the right DLFC. This region seems to be involved in wide, bilateral frontotemporal and limbic metabolic networks that are potentiated in HE prodromal AD patients not only with respect to LE prodromal AD patients but also with respect to $\mathrm{HE}$ controls.

The evidence of more AD-typical brain hypometabolism in $\mathrm{HE}$ than in LE prodromal AD patients expressing the same level of cognitive symptoms supports the existence of cognitive reserve. However, this finding cannot provide clues about the functional mechanisms underlying cognitive reserve. Two mechanisms have been described as being part of cognitive reserve, that is, neural reserve and neural compensation (11). Neural reserve reflects preexisting brain networks that are more efficient or have greater capacity and may therefore be less susceptible to disruption, whereas neural compensation refers to the adoption of new, compensatory brain networks after disease has affected those networks typically used for particular tasks.

We directly addressed this latter aspect by investigating whether relatively higher metabolism is found in HE than in LE prodromal AD patients. Such a finding was indeed present in the right inferior, middle, and superior frontal gyri. This finding suggests that metabolic activity within the right DLFC — which is likely to be relatively spared by the disconnection process-can be increased through synaptic trafficking only in $\mathrm{HE}$ prodromal AD patients. This result is in keeping with previous studies demonstrating a positive impact of cognitive reserve-related life activity on gray matter densities in the middle frontal cortex (26). Similarly, functional MR imaging during a memory task showed that education positively correlated with frontal activity in healthy elderly individuals but not in young individuals (27). This finding suggests that the frontal cortex might be

TABLE 4

Results of Interregional Correlation Analysis of ROI Depression in LE and HE Prodromal AD Patients

\begin{tabular}{|c|c|c|c|c|c|c|c|c|c|}
\hline \multirow[b]{3}{*}{ Analysis } & \multirow{2}{*}{\multicolumn{3}{|c|}{ Cluster level }} & \multicolumn{6}{|c|}{ Peak level } \\
\hline & & & & \multirow{2}{*}{$\begin{array}{c}\text { Maximum } \\
\text { z score }\end{array}$} & \multicolumn{3}{|c|}{$\begin{array}{c}\text { Talairach } \\
\text { coordinates }\end{array}$} & \multirow[b]{2}{*}{ Cortical region } & \multirow[b]{2}{*}{$\mathrm{BA}$} \\
\hline & Cluster extent & Corrected $P$ & Cortical region & & $x$ & $y$ & $z$ & & \\
\hline \multirow[t]{6}{*}{ LE } & 731 & 0.045 & L occipital & 3.72 & -48 & -77 & 9 & Middle occipital gyrus & 19 \\
\hline & & & $\mathrm{L}$ temporal & 3.68 & -57 & -64 & 5 & Middle temporal gyrus & 37 \\
\hline & & & $\mathrm{L}$ temporal & 3.61 & -42 & -51 & -11 & Fusiform gyrus & 37 \\
\hline & & & R occipital & 3.50 & 4 & -86 & 34 & Cuneus & 19 \\
\hline & & & R occipital & 3.44 & 6 & -96 & 23 & Cuneus & 18 \\
\hline & & & R occipital & 3.22 & 20 & -74 & 33 & Cuneus & 7 \\
\hline \multirow[t]{4}{*}{$\mathrm{HE}$} & 678 & 0.03 & $\mathrm{~L}$ temporal & 4.32 & -61 & -62 & -5 & Inferior temporal gyrus & 37 \\
\hline & & & $\mathrm{L}$ temporal & 3.88 & -50 & -76 & -3 & Inferior temporal gyrus & 19 \\
\hline & & & $\mathrm{L}$ temporal & 3.64 & -48 & -73 & 17 & Middle temporal gyrus & 39 \\
\hline & & & L occipital & 3.30 & -44 & -76 & 26 & Superior occipital gyrus & 19 \\
\hline
\end{tabular}

$P$ values of $<0.001$ (uncorrected at voxel level) and $<0.05$ (FDR-corrected for multiple comparisons at cluster level) were accepted as statistically significant. In cluster level section, reported for each statistically significant cluster are number of voxels, corrected $P$ value, and cortical region where cluster was found. In peak level section, reported for each significant cluster are $z$ score, peak coordinates, corresponding cortical region, and BA. 

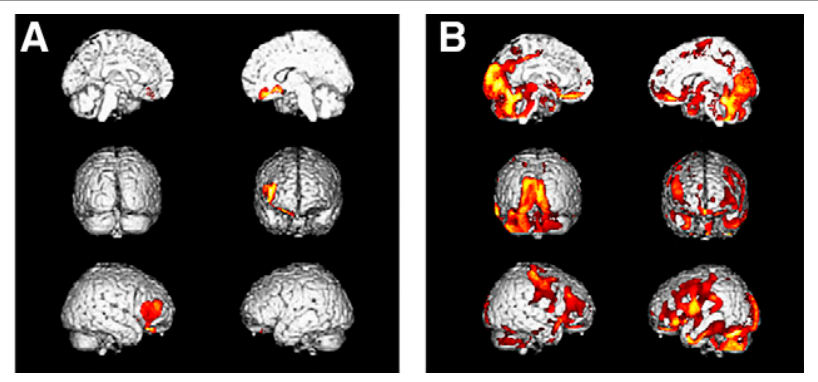

FIGURE 4. Interregional correlation analysis of ROI compensation in LE (A) and HE (B) prodromal AD patients. Talairach coordinates and further details are provided in Table 5.

engaged to support cognitive function even in the older individuals. Accordingly, some models of cognitive reserve propose frontal lobe activity (functional MR imaging) as a main compensatory mechanism already in place in HE elderly subjects. In the field of $\mathrm{AD}$, the topography of our results is, interestingly, in keeping with the emerging role of the DLFC in the discrimination between those MCI patients rapidly converting to dementia of Alzheimer type and those remaining stable $(16,28)$. Moreover, brain SPECT perfusion of the right DLFC has been shown to be negatively correlated in prodromal AD with hippocampal and parahippocampal MR imaging atrophy, suggesting a functional compensation in response to the lesional impairment of the medial temporal lobe (29).

However, other studies including both healthy elders and AD patients obtained different results, as they showed that premorbid intellectual ability was inversely correlated with cerebral metabolism in the prefrontal and premotor cortices (15). This difference in the directionality of the relationship between DLFC metabolism and cognitive reserve may be due to the earlier stage of the disease in the present group of prodromal AD patients. Our findings were obtained at the prodromal (amnestic MCI) stage of $\mathrm{AD}$ and thus highlight those compensatory mechanisms that are particularly active

TABLE 5

Results of Interregional Correlation Analysis of ROI Compensation in LE and HE Prodromal AD Patients

\begin{tabular}{|c|c|c|c|c|c|c|c|c|c|}
\hline \multirow[b]{3}{*}{ Analysis } & \multirow{2}{*}{\multicolumn{3}{|c|}{ Cluster level }} & \multicolumn{6}{|c|}{ Peak level } \\
\hline & & & & \multirow{2}{*}{$\begin{array}{c}\text { Maximum } \\
z \text { score }\end{array}$} & \multicolumn{3}{|c|}{$\begin{array}{l}\text { Talairach } \\
\text { coordinates }\end{array}$} & \multirow[b]{2}{*}{ Cortical region } & \multirow[b]{2}{*}{$\mathrm{BA}$} \\
\hline & Cluster extent & Corrected $P$ & Cortical region & & $x$ & $y$ & $z$ & & \\
\hline \multirow[t]{9}{*}{ LE } & 1,286 & 0.026 & $\mathrm{R}$ frontal & 4.31 & 22 & 28 & -18 & Middle frontal gyrus & 11 \\
\hline & & & $\mathrm{R}$ frontal & 4.23 & 30 & 30 & -15 & Inferior frontal gyrus & 47 \\
\hline & & & R frontal & 4.12 & 18 & 17 & -11 & Subcallosal gyrus & 47 \\
\hline & & & R frontal & 4.1 & 52 & 28 & 6 & Inferior frontal gyrus & 45 \\
\hline & & & $\mathrm{R}$ frontal & 3.97 & 42 & 49 & 16 & Middle frontal gyrus & 46 \\
\hline & & & $\mathrm{R}$ frontal & 3.95 & 48 & 29 & -2 & Inferior frontal gyrus & 45 \\
\hline & & & $R$ frontal & 3.9 & 46 & 41 & 4 & Inferior frontal gyrus & 46 \\
\hline & & & $R$ frontal & 3.87 & 38 & 31 & -12 & Inferior frontal gyrus & 47 \\
\hline & & & R limbic & 3.81 & 8 & 34 & -12 & Anterior cingulate & 32 \\
\hline \multirow[t]{18}{*}{$\mathrm{HE}$} & 11,303 & 0.0001 & $L$ frontal & 17.2 & -50 & 3 & 13 & Precentral gyrus & 44 \\
\hline & & & L occipital & 16 & -16 & -68 & 2 & Lingual gyrus & 18 \\
\hline & & & $\mathrm{R}$ frontal & 15.9 & 30 & -13 & 47 & Middle frontal gyrus & 6 \\
\hline & & & R frontal & 15.6 & 36 & -8 & 37 & Precentral gyrus & 6 \\
\hline & & & $\mathrm{R}$ frontal & 14.3 & 22 & 16 & 49 & Superior frontal gyrus & 8 \\
\hline & & & L limbic & 8.84 & -18 & -47 & -4 & Parahippocampal gyrus & 19 \\
\hline & & & L temporal & 8.22 & -40 & 8 & -31 & Superior temporal gyrus & 38 \\
\hline & & & L temporal & 5.61 & -61 & -37 & -8 & Middle temporal gyrus & 21 \\
\hline & & & L temporal & 5.39 & -63 & -24 & -21 & Inferior temporal gyrus & 20 \\
\hline & & & L parietal & 5.12 & -20 & -46 & 52 & Precuneus & 7 \\
\hline & & & L parietal & 4.96 & -18 & -47 & 36 & Precuneus & 31 \\
\hline & & & $\mathrm{R}$ temporal & 4.90 & 55 & -21 & -23 & Fusiform gyrus & 20 \\
\hline & & & $\mathrm{R}$ temporal & 4.80 & 59 & -11 & -18 & Inferior temporal gyrus & 21 \\
\hline & & & R frontal & 4.78 & 10 & 54 & 21 & Superior frontal gyrus & 9 \\
\hline & & & $\mathrm{R}$ frontal & 4.4 & 22 & 33 & 37 & Middle frontal gyrus & 8 \\
\hline & & & L sublobar & 3.96 & -30 & 10 & -2 & Claustrum & \\
\hline & & & R temporal & 3.78 & 48 & 17 & -13 & Superior temporal gyrus & 38 \\
\hline & 2,000 & 0.003 & L cerebellum & 14.6 & -36 & -54 & -36 & Cerebellar tonsil & \\
\hline
\end{tabular}

$P$ values of $<0.001$ (uncorrected at voxel level) and $<0.05$ (FDR-corrected for multiple comparisons at cluster level) were accepted as statistically significant. In cluster level section, reported for each statistically significant cluster are number of voxels, corrected $P$ value, and cortical region where cluster was found. In peak level section, reported for each significant cluster are $z$ score, peak coordinates, corresponding cortical region, and BA. 


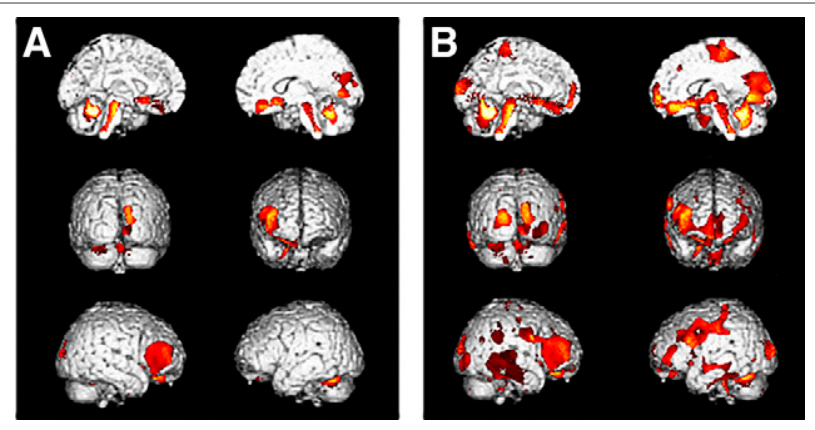

FIGURE 5. Interregional correlation analysis of ROI compensation in LE (A) and HE (B) controls. Other figure details are provided in legend to Figure 2. Talairach coordinates and further details are provided in Supplemental Table 3.

at this stage of the disease but may fail at subsequent stages. It is likely that compensatory mechanisms in the DLFC are still functionally active in the predementia stage and that their failure could be one of the pathophysiologic correlates of the conversion to AD dementia (30).

However, cognitive reserve in prodromal AD patients is likely based on networks, rather than on individual areas, that have been investigated by means of cognitive activation studies using $\mathrm{H}_{2}{ }^{15} \mathrm{O}$ PET and functional MR imaging $(11,12)$. This approach relies on the hypothesis that cognitive reserve begins either with the activation of networks typically used to accomplish a given task (the so-called neural reserve) (31) or with the recruitment of alternative compensatory networks, which would suggest neural compensation. Since this approach focuses on the networks that directly underlie task performance, it does not address the alternative concept of how cognitive reserve is, more generally, functionally mediated. In fact, one can hypothesize that cognitive reserve is mediated by non-task-specific networks that can be recruited during several less specific tasks (32). This view is supported by the fact that cognitive reserve helps maintain effective function across a wide range of activities despite the presence of brain pathology. Our approach thus aimed to disclose these resting networks by analyzing metabolic connectivity in HE and LE prodromal AD with a similar severity of cognitive impairment despite greater brain metabolic damage in the HE group. In HE prodromal AD, metabolism in the DLFC showed wide correlations with several cortical areas in both hemispheres (frontal, temporal, and occipital cortex; parahippocampal gyrus; and precuneus), as well as in the cerebellar hemispheres, but was just substantially autocorrelated in LE prodromal AD. Without entering into the detailed meaning of each correlation site, it is worth noting that these sites involved mainly those areas that were not affected by significant hypometabolism, as shown in Figure 1A, and thus were more functionally preserved. From the methodologic point of view, it could be argued that a more generalized relative brain hypometabolism in HE prodromal AD could have been responsible for this wider interregional correlation.
However, several brain regions included in these metabolic networks did not show significant hypometabolism with respect to both control and LE prodromal AD patients. Even more important, the analysis of the metabolic correlation of ROI depression indeed showed just autocorrelation both in the HE and in the LE prodromal AD groups. Finally, in a previous metabolic connectivity study (17), we demonstrated that the general population of prodromal AD patients has reduced metabolic connectivity with respect to cognitively normal controls both in metabolically impaired and non-metabolically impaired brain regions. Although based on a different methodology, the present findings align nicely with those of the few available activation studies aiming to disclose common or task-specific networks for cognitive reserve. By means of functional MR imaging, Stern et al. (32) investigated the presence of a single network as a likely candidate for a generic cognitive reserve network, through the demonstration of common sites of activation as a function of cognitive reserve, across 2 tasks with different cognitive processing demands (encoding and retrieval of a nonverbal serial recognition task). This common network was shown to be centered in the right and left superior frontal (BA 10), left medial frontal (BA 9), right medial frontal (BA 6 and 8), and left middle frontal (BA 8) gyri. The above-discussed metabolic connectivity analyses suggested explanations about how $\mathrm{HE}$ prodromal $\mathrm{AD}$ patients can cope better with a more severe AD-related hypometabolic involvement. However, the analyses did not completely elucidate whether these metabolic networks are due to preserved functional connections that are physiologically present in more educated subjects (i.e., the brain reserve component of cognitive reserve) or to the recruitment of alternative neural networks able to support cognitive function just in the presence of diseaserelated damage elsewhere (i.e., the brain compensation component of cognitive reserve). To specifically investigate this aspect, the metabolic connectivity of the right DLFC was tested in both HE controls and LE controls. In the post hoc analysis, we demonstrated that the DLFCrelated wide metabolic network in HE prodromal AD patients was topographically similar to but quantitatively more pronounced than that in LE prodromal AD patients

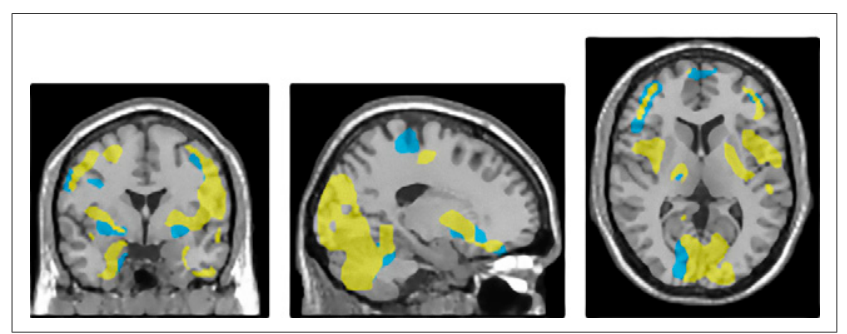

FIGURE 6. Point-by-point comparison of distribution of results of voxelwise interregional correlation analysis of $\mathrm{ROI}$ compensation in $\mathrm{HE}$ prodromal $\mathrm{AD}$ patient and $\mathrm{HE}$ control. Brain regions that significantly correlated with $\mathrm{ROI}$ compensation are superimposed on MR scan (yellow in HE prodromal AD patient and blue in HE control). 
and even HE controls. Some areas correlating with the right DLFC just in HE prodromal $\mathrm{AD}$, such as the cerebellum and superior temporal-parietal cortex, seem to be exquisitely related to neural compensation (Fig. 6). This result suggests that both mechanisms, neural reserve and neural compensation, are activated in HE prodromal AD.

There were some limitations to our study. First, we tested education as a proxy of cognitive reserve but did not evaluate the combined effect of occupation or the possible confounding effect of apolipoprotein E genotype, as these data were available for just some of the patients. Second, the use of different PET scanners is a potential limitation of this study, although we tried to minimize any such limitation by using a multiple-factor approach, including the balanced number of patients and controls among centers and the covariation for the recording center as a nuisance variable in SPM analyses.

\section{CONCLUSION}

This study confirmed greater brain metabolic damage in the AD-typical posterior limbic and associative cortex in HE prodromal AD patients. Besides confirming the existence of cognitive reserve in prodromal $\mathrm{AD}$, the present findings provide new insight into the functional mechanisms that mediate cognitive reserve by identifying higher metabolic activity in the DLFC. Moreover, the findings show more extended and significant correlations of metabolism within the right DLFC with other brain regions in HE than in LE prodromal AD patients or even HE controls. These data suggest that the phenomenic construct of cognitive reserve in $\mathrm{HE}$ prodromal $\mathrm{AD}$ patients is a result of both neural reserve and neural compensation.

\section{DISCLOSURE}

The costs of publication of this article were defrayed in part by the payment of page charges. Therefore, and solely to indicate this fact, this article is hereby marked "advertisement" in accordance with 18 USC section 1734. No potential conflict of interest relevant to this article was reported.

\section{REFERENCES}

1. Stern Y. What is cognitive reserve? Theory and research application of the reserve concept. J Int Neuropsychol Soc. 2002;8:448-460.

2. McDowell I, Xi G, Lindsay J, et al. Mapping the connections between education and dementia. J Clin Exp Neuropsychol. 2007;29:127-141.

3. Ngandu T, von Strauss E, Helkala EL, et al. Education and dementia: what lies behind the association? Neurology. 2007;69:1442-1450.

4. Mungas D, Reed BR, Jagust WJ, et al. Volumetric MR imaging predicts rate of cognitive decline related to $\mathrm{AD}$ and cerebrovascular disease. Neurology. 2002;59:867-873.

5. Chui HC, Zarow C, Mack WJ, et al. Cognitive impact of subcortical vascular and Alzheimer's disease pathology. Ann Neurol. 2006;60:677-687.

6. Bennett DA, Schneider JA, Arvanitakis Z, et al. Neuropathology of older persons without cognitive impairment from two community-based studies. Neurology. 2006;66:1837-1844.

7. Driscoll I, Resnick SM, Troncoso JC, et al. Impact of Alzheimer's pathology on cognitive trajectories in nondemented elderly. Ann Neurol. 2006;60:688-695.
8. Nucci M, Mapelli D, Mondini S. The cognitive reserve questionnaire (cognitive reserveIq): a new instrument for measuring the cognitive reserve. Aging Clin Exp Res. 2012;24:218-226.

9. Reed BR, Mungas D, Farias ST, et al. Measuring cognitive reserve based on the decomposition of episodic memory variance. Brain. 2010;133:2196-2209.

10. Kemppainen NM, Aalto S, Karrasch M, et al. Cognitive reserve hypothesis: Pittsburgh compound B and fluorodeoxyglucose positron emission tomography in relation to education in mild Alzheimer's disease. Ann Neurol. 2008;63: $112-118$.

11. Stern Y, Habeck C, Moeller J, et al. Brain networks associated with cognitive reserve in healthy young and old adults. Cereb Cortex. 2005;15:394-402.

12. Bosch B, Bartrés-Faz D, Rami L, et al. Cognitive reserve modulates task-induced activations and deactivations in healthy elders, amnestic mild cognitive impairment and mild Alzheimer's disease. Cortex. 2010;46:451-461.

13. Garibotto V, Borroni B, Kalbe E, et al. Education and occupation as proxies for reserve in aMCI converters and AD: FDG-PET evidence. Neurology. 2008;71: 1342-1349.

14. Perneczky R, Drzezga A, Diehl-Schmid J, et al. Schooling mediates brain reserve in Alzheimer's disease: findings of fluoro-deoxy-glucose-positron emission tomography. J Neurol Neurosurg Psychiatry. 2006;77:1060-1063.

15. Alexander GE, Furey ML, Grady CL, et al. Association of premorbid intellectual function with cerebral metabolism in Alzheimer's disease: implications for the cognitive reserve hypothesis. Am J Psychiatry. 1997;154:165-172.

16. Mosconi L, Pupi A, De Cristofaro MT, et al. Functional interactions of the entorhinal cortex: an ${ }^{18} \mathrm{~F}$-FDG PET study on normal aging and Alzheimer's disease. J Nucl Med. 2004;45:382-392.

17. Morbelli S, Drzezga A, Perneczky R, et al. Resting metabolic connectivity in prodromal Alzheimer's disease: a European Alzheimer Disease Consortium (EADC) project. Neurobiol Aging. 2012;33:2533-2550.

18. Hughes CP, Berg L, Danziger WL, et al. A new clinical scale for the staging of dementia. Br J Psychiatry. 1982;140:566-572.

19. Petersen RC. Mild cognitive impairment as a diagnostic entity. J Intern Med. 2004;256:183-194.

20. McKhann G, Drachman D, Folstein M, et al. Clinical diagnosis of Alzheimer's disease: report of the NINCDS-ADRDA Work Group under the auspices of Department of Health and Human Services Task Force on Alzheimer's Disease. Neurology. 1984;34:939-944.

21. American Psychiatric Association. Diagnostic and Statistical Manual of Mental Disorders: DSM-IV-TR. 4th ed., text revision. Washington, DC: American Psychiatric Association; 2000.

22. Varrone A, Asenbaum S, Vander Borght T, et al. EANM procedure guidelines for PET brain imaging using $\left[{ }^{18} \mathrm{~F}\right] \mathrm{FDG}$, version 2. Eur J Nucl Med Mol Imaging. 2009;36:2103-2110.

23. Friston KJ, Holmes AP, Worsley KJ, et al. Statistical parametric maps in functional imaging: a general linear approach. Hum Brain Mapp. 1994;2:189-210.

24. Gispert JD, Pascau J, Reig S, et al. Influence of the normalization template on the outcome of statistical parametric mapping of PET scans. Neuroimage. 2003;19: $601-612$.

25. Lee DS, Kang H, Kim H, et al. Metabolic connectivity by interregional correlation analysis using statistical parametric mapping (SPM) and FDG brain PET: methodological development and patterns of metabolic connectivity in adults. Eur J Nucl Med Mol Imaging. 2008;35:1681-1691.

26. Rovio S, Spulber G, Nieminen LJ, et al. The effect of midlife physical activity on structural brain changes in the elderly. Neurobiol Aging. 2010;31:1927-1936.

27. Springer MV, McIntosh AR, Winocur G, et al. The relation between brain activity during memory tasks and years of education in young and older adults. Neuropsychology. 2005;19:181-192.

28. Mosconi L, Perani D, Sorbi S, et al. MCI conversion to dementia and the APOE genotype: a prediction study with FDG-PET. Neurology. 2004;63:2332-2340.

29. Guedj E, Barbeau EJ, Didic M, et al. Effects of medial temporal lobe degeneration on brain perfusion in amnestic MCI of AD type: deafferentation and functional compensation? Eur J Nucl Med Mol Imaging. 2009;36:1101-1112.

30. Nobili F, Salmaso D, Morbelli S, et al. Principal component analysis of FDG PET in amnestic MCI. Eur J Nucl Med Mol Imaging. 2008;35:2191-2202.

31. Scarmeas N, Zarahn E, Anderson KE, et al. Cognitive reserve modulates functional brain responses during memory tasks: a PET study in healthy young and elderly subjects. Neuroimage. 2003;19:1215-1227.

32. Stern Y, Zarahn E, Habeck C, et al. A common neural network for cognitive reserve in verbal and object working memory in young but not old. Cereb Cortex. 2008;18:959-967. 